Revista de Psicología de la PUCP. Vol. XXI, 1, 2003 Edición de aniversario

\title{
La Escala Superlativa S de Butcher y Han (1995): el fingimiento en la adaptación española del MMPI-2
}

\author{
Guadalupe Sánchez Crespo y Fernando Jiménez Gómez' \\ Universidad de Salamanca
}

\begin{abstract}
Se investigó la conducta de fingir en la adaptación española del MMPI-2. Participaron 3.035 personas, de los cuales los grupos "normal" (1.723) y "clínico" (561) respondieron con sinceridad al cuestionario. A un grupo de 269 personas "normales" se les instruyó para que muestren intencionadamente una imagen negativa de sí mismos (grupo "mala imagen") y a otros 282 , para que mostraran una imagen positiva (grupo "buena imagen"); otros 200 participantes contestaron al cuestionario sin atender a la información contenida en los ítems (grupo "repuestas inconsistentes"). Los resultados mostraron la sensibilidad de la Escala para detectar a aquellos que falsean sus respuestas presentando una imagen más favorable. Se observaron dificultades para discriminar a los que contestan sin tener en cuenta a los ítems.
\end{abstract}

Palabras clave: Escala Superlativa S, simulación, buena imagen.

The Superlative Scale S of Butcher and Han (1995): the "fake-good" in the Spanish adaptation of the MMPI-2

The present study investigated the "fake-good" behaviour using the Superlative Scale $S$ in the Spanish adaptation of the MMPI-2. From the 3.035 participants, the "normal" group (1.723) and the "clinic" group (561) responded with sincerity to the questionnaire. A group of 269 "normal" participants was asked to show a negative image of themselves (group "fake-bad") and other 282 participants, to show a positive image (group "Fake-good"); finally a group of 200 participants answered to the questionnaire without following the information of the items (group "inconsistent answers"). The results showed the sensibility of this to detect the individuals that falsify their answers trying to present their more positive image. There were difficulties to identify individuals who answered to the questionnaire without keeping in mind the information of the items.

Key words: Superlative Scale S, positive self, fake-good.

Profesores de la Universidad de Salamanca. Facultad de Psicología. Dpto. Personalidad, Evaluación y Tratamiento Psicológicos. Correo electrónico: fjimenez@usal.es y lupes@usal.es. 

A Lucho ${ }^{2}$, de sus amigos, a quienes no nos dejó disfrutar todo lo que hubiéramos querido.

Para considerar un protocolo del MMPI-2 como aceptable el sujeto debe leer y considerar el contenido de cada ítem y responder a ellos según considere que, aplicado a sí mismo resulten ser "verdadero" o "falso". Ocasionalmente el individuo puede responder de una forma idiosincrásica teniendo en cuenta, o no, el contenido informativo de los ítems que forman el cuestionario. Tal comportamiento puede ocurrir entre personas que carecen de las adecuadas destrezas de lectura y/o comprensión, pueden encontrarse confusos ante tal tarea o pueden presentar una actitud negativa hacia los procedimientos de evaluación o hacia los mismos profesionales de la Psicología. Otras veces los sujetos se pueden encontrar altamente motivados para ofrecer al evaluador un buen ajuste psicológico ante las preguntas formuladas en el MMPI-2, intentando, con ello, ofrecer la mejor imagen de sí mismos.

En circunstancias ideales, el examinador debería poder valorar la tendencia a responder por parte del sujeto intentando asegurar que los sujetos completen la prueba con la honestidad y sinceridad que señalan las instrucciones del test. Por ello es importante poder apreciar la disposición y motivación del sujeto y saber, por parte del profesional administrador, cómo detectar los protocolos sospechosos de validez. Así, ante la sospecha de un protocolo que podría ser falsificado, y con las consecuencias correspondientes en su interpretación, sería muy útil al evaluador, poder detectar esta conducta del indivi-

2 Dr. Luis Trelles Montero, Profesor Principal del Departamento de Humanidades-Sección Psicología (3 de setiembre de 1942-23 de febrero del 2001). 
duo al realizar la prueba. La falta de congruencia entre el perfil y la conducta observada puede aportarnos alguna idea del tipo, clase o estilo de respuesta que da el sujeto.

Si un protocolo del MMPI-2 se juzgase nulo o invalidado, el examinador debería estar dispuesto a hablar de la situación con el sujeto y volver, si es posible, a administrarle la prueba. Frecuentemente, una segunda vez produce un protocolo válido. Si esto no fuera posible o siguiera dando un protocolo no válido, la interpretación ha de suspenderse. Según Graham (1990), sería tentador interpretar el protocolo de una persona que se presenta con buena imagen, negando, ocultando o rechazando un número de síntomas y problemas clínicos importantes, siendo realmente una persona inadaptada o desajustada. Tal conclusión, sigue comentando Graham (1990) no está justificada. La persona puede estar bien ajustada pero por circunstancias diversas, siente la necesidad de presentarse mejor adaptada. Este tipo de casos no es extraño encontrarlo en ámbitos jurídicos y sobre todo en progenitores que intentan conseguir la guardia y custodia de sus hijos o la adopción (Jiménez y Sánchez, 2003).

El modelo "adaptacional" de Rogers (1990), asume que el sujeto percibe la evaluación como un reto en que el mismo sujeto tiene algo que ganar o perder dependiendo de los resultados de la evaluación. El fingimiento o simulación se convierte en una conducta de refuerzo y que puede resultar potencialmente útil.

Algunos usuarios y evaluadores profesionales del MMPI- 2 consideran un protocolo nulo, y por consiguiente no interpretable, cuando tiene más de 30 ítems sin contestar u omitidos o la puntuación $T$ es mayor de 70 en una o más de las escalas estándar de validez. Aunque esta es una práctica muy prudente no deja de ser una visión muy simplista de resolver el problema. Así, por ejemplo, en el manual del MMPI-2 (Butcher, Dahistrom, Graham, Tellegen y Kaemmer, 1989) se presentan casos en los que la puntuación de la escala $\mathrm{F}$, encontrándose 
en un rango de puntuación entre 71 a 90, puede estar indicando una psicosis. Gynther, Altman y Warbin (1973) demostraron que perfiles con puntuaciones $T=100$ en la escala $F$ pueden estar plenamente correlacionados con algunos comportamientos y tipos de personalidad clínicos (desorientaciones, alucinaciones, ilusiones, escasa atención). Igualmente decir, la experiencia clínica nos demuestra que encontrar una escala $\mathrm{K}$ con puntuaciones $T>70$ entre las personas con buen nivel educativo no es algo excepcional.

Los indicadores de la validez que disponemos en el MMPI-2 deben ser analizados de forma combinada, determinando la peculiaridad de cada variable y las implicaciones posibles cuando se conjugan con los resultados de las demás. Vamos a analizar y comparar en nuestro estudio los resultados obtenidos de la Escala Superlativa S de Butcher y Han (1995) con las escalas tradicionales y adicionales de validez del MMPI-2; es decir, con la escala L (mentira), la escala $F$ (infrecuencia), la escala $\mathrm{Fb}$ (F posterior), la escala $\mathrm{K}$ (corrección), la escala TRIN (Inconsistencia de las respuestas verdadero) y la escala VRIN (Inconsistencia de respuestas variables).

La Escala Superlativa S de Butcher y Han (1995) fue elaborada para evaluar la presentación de sí mismo de una manera muy bien ajustada psicológicamente. Un primer estudio lo realizaron estos autores (Butcher y Han, 1995, p.25) para seleccionar a varones aspirantes a pilotos de líneas aéreas y se contrastaron sus resultados con 1.138 varones de la muestra que sirvió para tipificar esta prueba. Sus ítems significativos diferencialmente fueron seleccionados cuando su contribución a la consistencia interna de la prueba resultó ser aceptable. De estos análisis se diferenciaron cinco subescalas a partir del análisis factorial realizado con 50 ítems: creencia en la bondad humana (S1, 15 ítems), Serenidad (S2, 13 ítems), Satisfacción con la vida (S3, 8 ítems), Negación de la ira o irritabilidad (S4, 8 ítems) y Negación de defectos (S5, 5 ítems). 
Nuestro estudio-intenta aportar alguna luz a estas investigaciones utilizando el formato general $(S)$ de esta escala (sin referencia a las subescalas) con una variada muestra española importante $(N=$ 3.035), dividida en diferentes grupos, analizando y comparando sus resultados. A un grupo de participantes en esta investigación se les ha instruido para completar el cuestionario del MMPI-2 para que intencionadamente mostraran su mejor imagen y a otros para que, de la misma manera, mostraran su imagen negativa.

\section{Objetivos}

Nuestro objetivo general intenta analizar la sensibilidad de la Escala Superlativa S de Butcher y Han (1995) con una muestra española para poder discriminar entre protocolos contestados de forma honesta y aquellos en los que se ha realizado algún tipo de distorsión en las respuestas de los sujetos.

Nuestros objetivos específicos se centran en poder detectar el tipo de distorsión realizada. Estos tipos de distorsión vienen especificada de tres formas:

- Poder detectar e identificar perfiles de exageración o minimización en sus respuestas.

- Poder diferenciar e identificar perfiles que expresen buena imagen o fingimiento positivo.

- Poder aportar la colaboración diagnóstica de otras escalas para una adecuada interpretación conjunta.

\section{Hipótesis}

Estas tres formas de distorsión se van a concretar en un objetivo más preciso y específico que conforman nuestra hipótesis de investigación: Las personas que contestan a los ítems MMPI-2 fingiendo te- 
ner características positivas obtendrán valores significativos más altos en la Escala Superlativa $\mathrm{S}$ de aquellos otros que no lo hacen.

\section{Metodología}

\section{Participantes}

Se utilizó una gran parte de la muestra empleada en la adaptación castellana (Avila y Jiménez, 1999) para nuestro estudio, especialmente la muestra clínica y gran parte de la población normal. Tanto las muestras de "buena imagen", "mala imagen" como la que ofreció un variado repertorio de respuestas inconsistentes fueron específicamente obtenidas teniendo muy claro el objetivo de este estudio. Por razones obvias se rechazaron todos aquellos protocolos que presentaran una edad superior a los 64 años y también aquellos que presentaran una puntuación en "No sé / no puedo contestar (“?”) = 30 .

Los administradores de la prueba son profesionales de la psicología, con más de 10 años de experiencia, conocedores, ampliamente, de la técnica del MMPI. La muestra de esta investigación se recogió en la casi totalidad de las diferentes comunidades de nuestra geografía española quedando distribuidas por género y especificadas en el Cuadro 1.

\section{Cuadro 1}

Participantes según grupos y género

\begin{tabular}{|c|c|c|c|c|c|c|c|}
\hline Normal & \multicolumn{2}{|c|}{ Clínica } & \multicolumn{2}{|c|}{ B. Imagen } & \multicolumn{2}{|c|}{ M. Imagen } & Rptas. Inconsist. \\
\hline Varones Mujeres & Varones & Mujeres & Varones & Mujeres & Varones & Mujeres & Varones Mujeres \\
\hline 1039 & 252 & 309 & 119 & 163 & 97 & 172 & 100 \\
\hline 1.723 & \multicolumn{2}{|c|}{561} & \multicolumn{2}{|c|}{282} & \multicolumn{2}{|c|}{269} & 200 \\
\hline
\end{tabular}

Los individuos participantes en nuestra investigación, denominados como "grupo normal" está compuesta por 1.723 personas consideradas 
como normales (sin evidencia de patología) que han contestado a los items del MMPI-2 de forma totalmente sincera y honesta (forma estándar). Estos sujetos presentan una media de edad de casi 30 años (29 años y 10 meses) de los cuales 1.039 son mujeres y 684 varones.

Los participantes denominados, en nuestra investigación, como "grupo clínico" son pacientes en régimen abierto ambulatorio que acuden a su Centro de Salud correspondiente, con diferentes problemas de tipo psicológico o psiquiátrico (de gravedad leve o moderada), solicitando la ayuda de un profesional de la psicología. Esta muestra no incluye pacientes crónicos internados en un centro psiquiátrico o con trastornos considerados de gravedad severa . El número de sujetos son de 561 pacientes, de los cuales 309 son mujeres y 252 son varones, teniendo una media de edad de 34 años.

Los individuos denominados, en nuestra investigación, como "grupo buena imagen" son personas consideradas "normales" ( $\sin$ evidencia de patología) a las que se les instruyó previamente para que llevaran a cabo la prueba del MMPI-2 intentando of recer una "buena imagen" de sí mismo. El número de sujetos fue de 282, de los cuales 163 fueron mujeres y 119 varones, teniendo una media de edad de 27 años.

Los sujetos denominados en nuestra investigación como de "grupo mala imagen" son individuos considerados "normales" (igualmente sin evidencia de patología) a los que también se les instruyó para que contestaran al MMPI-2 intentando ofrecer una "mala imagen" de sí mismo. El número de sujetos fue de 269, de los cuales 172 fueron mujeres y 97 varones, teniendo una media de edad de casi 27 años (26 años y 11 meses).

Por fin, se generaron manualmente el grupo de "Respuestas inconsistentes". Para ello se cumplimentaron 200 protocolos sin tener en cuenta el sentido de los ítems, independientes de la edad y sexo. 
Las respuestas han seguido estrategias diferentes como responder al cuestionario como Verdadero, como Falso, alternativamente Falso/Verdadero (cada 5, cada 10, cada columna de la hoja de respuesta, etc.).

\section{Instrumentos}

El instrumento único es el Cuestionario de Personalidad MMPI2, en su adaptación española (Avila y Jiménez, 1999).

En este estudio se tuvieron en cuenta para su análisis las escalas tradicionales de Validez L, F, $\mathrm{K}$ y las adicionales Fb, VRIN y TRIN.

Escala Superlativa S de Butcher y Han (1995)

La escala Superlativa S, o "auto-presentación superlativa" como la denomina Nichols, (2002) está compuesta por un total de 50 ítems con una proporción de Verdadero/Falso de 6/44 (ver Anexo) compartiendo 10 ítemes con la escala $\mathrm{K}$ con la que presenta una alta correlación en los estudios americanos (Dahlstron, Welsh y Dahlstron, 1975). Sus ítemes son menos sutiles, más abiertamente expresados y más deseables socialmente que los de la escala $\mathrm{K}$. La temática abordada por la Escala $S$ se encuentra referida por una benévola creencia en la virtud, en el honor y la nobleza de los demás, en la satisfacción con la propia vida y sus avatares e incluso de la serenidad, conformidad y ausencia de irritabilidad ante los eventos teóricamente estresantes para la mayoría de los individuos.

\section{Escala L (Mentira)}

Esta escala, denominada originalmente escala de "mentira", fue diseñada para detectar, de forma sencilla, los intentos, por parte de los sujetos, de presentarse a sí mismos de forma favorable. La escala se basa en la idea de que los individuos que intentan reclamar un excelente ajuste psicológico se atribuirán ítems que indican elevadas 
características morales, éticas y de responsabilidad. Los 15 ítems que componen esta escala son evidentes en su contenido y se centran sobre la afirmación de gran virtud (por ejemplo: "a veces tengo ganas de maldecir" (falso) o "no siempre digo la verdad" (falso). Las puntuaciones por encima de $65 \mathrm{~T}$ sugieren que el individuo se presenta de una forma positiva intentando crear una idea favorable y no realista de sus características morales y ajuste psicológico.

Esta escala es un buen indicador de respuesta distorsionada o inválida, encontrándose asociada también con características de personalidad que sugieren ingenuidad, pensamiento rígido y una imagen no realista. No es aconsejable la interpretación asilada de esta escala (ni ninguna del MMPI-2), ya que, justamente con la puntuación K (corrección) puede aportarnos matizaciones o clarificaciones de su hipótesis interpretativa.

\section{Escala F (Infrecuencia)}

Esta escala, originariamente, fue elaborada para detectar las formas anormales o atípicas de responder (Meehl y Hathaway, 1946; Mckinley, Hathaway y Meehl, 1948), como una medida de la tendencia a reconocer o admitir una extensa gama de problemas psicológicos o también para "fingirse malo" o crear una mala imagen. Un individuo que presenta puntuaciones altas en F está reconociendo un amplio rango de quejas o dolencias que son infrecuentes para la población general reflejando con ello una tendencia a la exageración de los problemas o una presencia real de patología. Los 64 ítems que componen la escala original fueron contestados en la dirección de puntuación por menos del $10 \%$ de los sujetos adultos de la población normalizada. Muchos de los ítems de la escala $\mathrm{F}$ fueron suprimidos del MMPI-2 porque su contenido podría ser molesto, dejando a la escala con 60 ítems. 


\section{Escala K (corrección, defensividad)}

La experiencia con la escala $L$ del MMPI pronto indicó que parecía ser totalmente insensible a la distorsión de algunos perfiles. La escala $\mathrm{K}$ fue elaborada como algo más sutil y efectiva ante los intentos del sujeto de negar o simular síntomas psicopatológicos deseando con ello presentarse de forma más favorable e incluso poder, en un sentido opuesto, exagerar su patología para evidenciar una mala imagen de sí mismo (Meehl y Hathaway, 1946; Mckinley y Hathaway, 1948). De esta manera las puntuaciones elevadas dadas en la escala $\mathrm{K}$ fueron consideradas como una forma de defensividad ante el abordaje del test, considerando que las puntuaciones bajas fueron pensadas para poder apreciar una inusual franqueza y autocrítica.

La escala $\mathrm{K}$ originariamente estaba compuesta por 30 ítemes que fueron empíricamente identificados contrastando las respuestas a los ítemes de sujetos patológicos que producían perfiles normales (falsos negativos) con las respuestas dadas por sujetos normales. La versión del MMPI-2 sigue manteniendo el mismo número de ítemes aunque uno de ellos fue cambiado. Los 30 ítems componentes de esta escala, cubren una amplia área de contenidos diversos en los que una persona puede llevar a cabo una negación de sus problemas (hostilidad, recelo, discordias familiares, ausencia de confianza, excesiva preocupación).

Los trabajos de reestructuración y reestandarización del MMPI realizadas por el equipo de la Universidad de Minnesota (Butcher, Dahlstrom, Graham, Tellegen y Kaemmer, 1989) aportaron tres nuevas escalas de validez en el MMPI-2 para ayudar en la evaluación de actitudes invalidantes: Fb, VRIN y TRIN.

\section{Escala Fb (F posterior)}

La escala Fb ("F Back scale") es un índice adicional de validez del test y originariamente fue desarrollada en la versión revisada del 
MMPI para detectar la posible desviación de las respuestas motivadas por la extensión de la prueba a través del cuadernillo experimental utilizado en la recogida de datos normativos del MMPI-2 (Butcher, Dahlstrom, Graham, Tellegen y Kaemer, 1989). Este procedimiento utilizado para desarrollar la escala $\mathrm{Fb}$ fue similar al usado con la elaboración de la escala $\mathrm{F}$ estándar. La escala $\mathrm{Fb}$ incluía originalmente 64 ítems, apareciendo posteriormente en la edición experimental del cuadernillo con un $10 \%$ de respuestas en la dirección de la puntuación cuando fue contestada por la población normal. La versión definitiva para el MMPI-2 de esta escala se quedó en 40 de los 64 ítemes originales.

En la versión definitiva del MMPI-2, el último ítem de la escala $\mathrm{F}$ es el 361, sin embargo el primer ítem de la escala $\mathrm{Fb}$ es el 281. Por lo tanto podemos decir que es fundamentalmente la segunda parte de la prueba la que detecta esta escala $\mathrm{Fb}$. No podemos afirmar, como algunos manuales indican, que es a partir del ítem 370. Esta escala Fb comparte un total de 7 ítemes con la escala Psiquiátrica Fp. Se correlaciona con otras escalas significativas de supervaloración en torno al 0,80 y al igual que ocurre con la escala $\mathrm{F}$, tiende a elevar la mayor parte de las escalas Clínicas básicas, especialmente con la Esquizofrenia (8.Sc), Psicastenia (7.Pt) Paranoia (6.Pa) y Desviación psicopática (4.Pd) (Nichols, 2002, p. 56).

\section{Escala VRIN (Inconsistencia de Respuestas Variables)}

Esta escala se elaboró para el MMPI-2 como un indicador adicional de validez (Butcher, Dahlstrom, Graham, Tellegen y Kaemer, 1989) con el objetivo de detectar la tendencia de los sujetos a responder de una forma inconsistente o incoherente a los ítemes. Esta escala VRIN se encuentra compuesta por 67 pares de ítemes (97 ítemes en total; 49 parejas distintas) con contenido similar u opuesto, cinco pares se corresponden con Verdadero-Verdadero y cinco con Falso-Falso solapándose con los de TRIN (Nichols, 2002). Cada vez 
que un sujeto responde a los ítemes en un par de forma incoherente o inconsistente se anotará un punto para la escala VRIN. Se produce incoherencia o inconsistencia si el sujeto contesta a los ítemes en la dirección propuesta.

Aunque las indicaciones de los autores (Butcher, Dahlstrom, Graham, Tellegen y Kaemmer, 1989) que llevaron a cabo la elaboración de esta escala nos advierten de su experimentalidad hasta encontrar datos empíricos más consistentes, no obstante, en sus investigaciones, sugieren que una puntuación directa igual o mayor a 13 (>14 para la adaptación española; Avila y Jiménez, 1999) nos puede estar indicando incoherencia o inconsistencia en sus respuestas y posiblemente puede estar invalidando los resultados de la prueba.

\section{Escala TRIN (Inconsistencia de las Respuestas Verdadero)}

La escala de Inconsistencia de Respuestas Verdadero (TRIN) fue desarrollada en el MMPI-2 con el propósito de poder identificar a los sujetos que responden de forma incoherente o inconsistente a los ítemes dando respuestas de "verdadero" de forma indiscriminada (aquiescencia, conformidad, consentimiento) o dando respuestas "falso" igualmente de forma indiscriminada, (no aquiescencia, disconformidad) (Butcher, Dahlstrom, Graham, Tellegen y Kaemmer, 1989). En estos casos el resultado de los perfiles deben ser invalidados y no interpretados.

Esta escala TRIN se compone de 23 pares de ítems (40 ítemes totales, 20 pares únicos) que son opuestos de contenidos. Dos respuestas "verdadero" para algún par de ítems o dos respuestas "falso" para otro par de ítemes indican inconsistencia o incoherencia en las respuestas. 


\section{Resultados}

Baer, Wetter y Berry (1995) encontraron que la escala S resultó ser efectiva para identificar a los estudiantes que contestaban intentando ofrecer una buena imagen. Para estos autores, el 92\% de los sujetos de "buena imagen" presentaban una puntuación directa $=29$. En nuestros resultados (Cuadro 2) podemos apreciar cómo el 90\% de estos mismos sujetos (aunque no son estudiantes), que igualmente intentan mostrar una buena imagen, presentan una puntuación directa $=$ 25. Los resultados no parecen ser muy dispares.

\section{Cuadro 2}

Análisis por grupos: Variable "S"y percentiles

\begin{tabular}{|cccccc|}
\hline Percentiles & Normal & Clínico & B. Imagen & M. Imagen & Rptas. Inconsist. \\
\hline $\mathbf{1 0}$ & $\mathbf{1 4}$ & 10 & 25 & 5 & 20 \\
$\mathbf{2 5}$ & 18 & 14 & 32 & 8 & 22 \\
$\mathbf{5 0}$ & 24 & 19 & 38 & 11 & 26 \\
$\mathbf{7 5}$ & 29 & 24 & 42 & 16 & 28 \\
$\mathbf{9 0}$ & 34 & 30 & 45 & 23 & 31 \\
\hline
\end{tabular}

E1 90\% de las 2.600 personas normales presentados por Butcher, Dahlstrom, Graham, Tellegen y Kaemer (1989) mostraron una puntuación directa $=13$ (nuestros resultados: $=14$ ). Cuando se trata de sujetos con graves trastornos, presentados por Greene (1997), el 90\% de los sujetos mostraron una puntuación directa $=8$ (nuestros clíni$\cos$, que no presentan una grave patología, $=10$ ).

Butcher y Han (1995) encontraron elevadas correlaciones (Graham, 2000, p. 36) con la escala K del MMPI-2 con muestras normales (.81 para varones; .92 para mujeres), y algo más bajas con la escala L (.46, para varones y .64 para mujeres). Si contemplamos el Cuadro 74, podemos ver que nuestros resultados son coincidentes, en todos los grupos analizados, con los de Butcher y Han (1995) con respecto a las variable $\mathrm{K}$ y $\mathrm{L}$, siendo más elevada en la primera. 
Semejantes resultados obtuvieron los investigadores Baer, Wetter, Nichols, Greene y Berry (1995) al llegar a la conclusión que los datos aportados por la escala $S$ añadidos a los de las escalas $L$ y $\mathrm{K}$ pueden discriminar entre personas que contestan a la prueba de forma honesta y sincera de aquellas que contestan de una forma pretendidamente perfecta y bien ajustadas psicológicamente.

\section{Cuadro 3}

Variable " $S$ ": Correlaciones".

\begin{tabular}{|cccccc|}
\hline Variables & Normal & Clínico & B. Imagen & M. Imagen & Rptas. Inconsist. \\
\hline L &. $\mathbf{4 5 1}$ & $\mathbf{. 4 5 0}$ & $\mathbf{. 7 3 2}$ & $\mathbf{. 7 3 9}$ & $\mathbf{. 4 4 0}$ \\
F & $\mathbf{. 4 7 6}$ & $\mathbf{- . 5 6 5}$ & $\mathbf{- . 5 5 9}$ & $\mathbf{. 3 7 4}$ & $\mathbf{. 3 3 4}$ \\
K & $\mathbf{. 8 1 3}$ & $\mathbf{. 8 0 9}$ & $\mathbf{. 8 4 5}$ &. $\mathbf{8 4 4}$ & $\mathbf{. 5 4 9}$ \\
Fb & $\mathbf{- . 4 6 1}$ & $\mathbf{- . 5 7 4}$ & $\mathbf{- . 5 0 7}$ & $\mathbf{- . 4 1 4}$ & $\mathbf{. 4 4 7}$ \\
VRIN & $\mathbf{- . 1 6 3}$ & .090 & $\mathbf{- . 5 4 8}$ & .591 & .134 \\
TRIN & $\mathbf{- . 2 0 3}$ & $\mathbf{- . 2 7 5}$ & -.121 & -.102 & $\mathbf{- . 4 4 9}$ \\
\hline
\end{tabular}

Nota $a^{\mathrm{a}}$ Los valores en negrita son estadísticamente significativos al $p<.001$.

Igualmente hemos podido apreciar en nuestro Cuadro 3, donde se exponen las diversas correlaciones entre los grupos y con las distintas variables, que existe un par de variables con las que se mantiene una notable y significativa correlación negativa: F, Fb. Lo cual nos lleva a poder apreciar la sensibilidad hacia el fingimiento positivo y de intentar ofrecer buena imagen más que el ofrecer el aspecto desfavorable de sí mismo.

Butcher y Han (1995) concluyen que las puntuaciones elevadas obtenidas en la escala $S$ son of recidas por personas no realistas, que informan positivamente de sí mismo, muy bien equilibrados psicológicamente y sin problemas. Pero estos investigadores no aportan dato alguno acerca de si esta variable puede detectar perfiles defensivos o perfiles de buena imagen (Graham, 2000). 
Guadalupe Sánchez Crespo y Fernando Jiménez Gómez

\section{Cuadro 4}

Variable "S": Puntuaciones Medias y DE Análisis comparativo entre grupos

\begin{tabular}{|c|c|c|c|c|c|c|c|c|}
\hline \multicolumn{2}{|c|}{ Normal } & \multicolumn{2}{|c|}{ Clínico } & \multicolumn{2}{|c|}{ Buena Imagen } & \multicolumn{3}{|c|}{ Mala Imagen Rptas. Inconsist. } \\
\hline Media & $\mathrm{DE}$ & Media & $\mathrm{DE}$ & Media & $\mathrm{DE}$ & Media & $\mathrm{DE}$ & Media DE \\
\hline 23.89 & 7.66 & 19.32 & 7.38 & 36.33 & 8.25 & 12.61 & 7.33 & $25.43 \quad 4.87$ \\
\hline
\end{tabular}

Consultando los Cuadros 4 y 5 confirmamos que es el grupo de buena imagen el que presenta las puntuaciones más elevadas, siendo estadísticamente significativa sus diferencias de puntuaciones medias con el resto de los grupos.

\section{Cuadro 5}

Variable "S": ANOVA- Diferencias significativas entre grupos

\begin{tabular}{|lrrc|}
\hline \multicolumn{1}{|c}{ Grupos } & Dif.medias & Dif. Crit. & $p$ \\
\hline Normal vs. Clínico & 4.58 & .71 & $<.0001$ \\
Normal vs. B. Imagen & -12.44 & .94 & $<.0001$ \\
Normal vs. M. Imagen & 11.28 & .96 & $<.0001$ \\
Normal vs. Rtas. Inc. & -1.54 & 1.10 & .0060 \\
Clínico vs. B. Imagen & -17.02 & 1.07 & $<.0001$ \\
Clínico vs. M. Imagen & 6.70 & 1.09 & $<.0001$ \\
Clínico vs. Rtas. Inc. & -6.11 & 1.21 & $<.0001$ \\
B. Imagen vs. M. Imagen & 23.72 & 1.25 & $<.0001$ \\
B. Imagen vs. Rtas. Inc. & 10.90 & 1.36 & $<.0001$ \\
M. Imagen vs. Rtas. Inc. & -12.82 & 1.37 & $<.0001$ \\
\hline
\end{tabular}

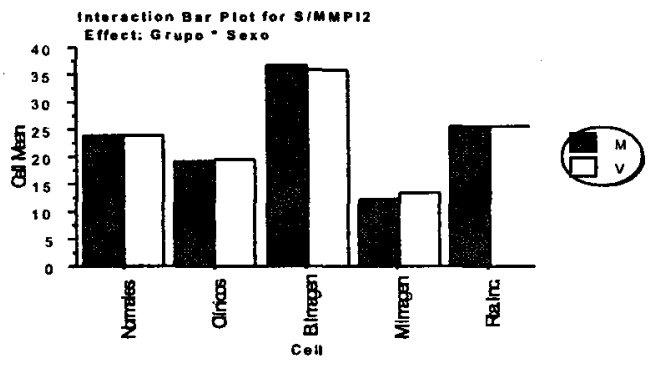


Figura 1. Variable "S". Análisis intersexos/intergrupos.

Si nos fijamos atentamente en el Cuadro 5, podemos apreciar que entre los grupos normales y los que contestan de forma inconsistente a los ítemes del cuestionario (Rptas. Inconsist.) fueron estadísticamente significativos cuando los sexos se analizaron conjuntamente, pero cuando realizamos el análisis separando los sexos (Cuadro 6) podemos apreciar que existen diferencias significativas entre estos dos grupos. Por esto mismo, a la hora de interpretar adecuadamente las variables y disponer de un criterio plenamente fiable, deberíamos acompañarnos de todas aquellas otras variables que correlacionan con ella de forma positiva y/o también negativa (las dos aportan información importante).

\section{Cuadro 6}

Variable "S": Diferencias intergrupos/intersexos"

\begin{tabular}{|c|c|c|c|c|c|c|c|c|c|}
\hline \multicolumn{5}{|c|}{$\begin{array}{l}\text { Fisters P SOlers } \\
\text { Effect. Grupos } \\
\text { Slgnfficance Level: } 5 \% \\
\text { Split By: Genero } \\
\text { Cell: M }\end{array}$} & \multicolumn{5}{|l|}{ 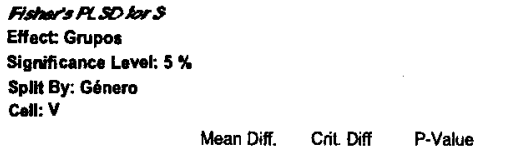 } \\
\hline B.Imagen, Clinicos & 17,460 & 1,387 & $<, 0001$ & $\mathbf{s}$ & \multirow{2}{*}{$\begin{array}{l}\text { B.Imagen, Clínicos } \\
\text { B.Imagen, M.Imagen }\end{array}$} & 16,423 & 1,689 & $<, 0001$ & S \\
\hline B.Imagen, M.Imagen & 24,479 & 1,566 & $<, 0001$ & $\mathbf{s}$ & & 22,525 & 2,077 & $<, 0001$ & s \\
\hline B.Imagen, Normales & 12,847 & 1,207 & $<, 0001$ & $\mathbf{s}$ & \multirow{2}{*}{$\begin{array}{l}\text { B.Imagen, Normales } \\
\text { B.Imagen, Rta.fnc. }\end{array}$} & 11,878 & 1.508 & $<.0001$ & $s$ \\
\hline B.Imagen, Rta.Inc. & 11,356 & 1,820 & $<, 0001$ & $\mathbf{s}$ & & 10,314 & 2,059 & $<.0001$ & $\mathrm{~s}$ \\
\hline Clinicos, M.Imagen & 7,019 & 1,363 & $<, 0001$ & $\mathbf{s}$ & \multirow[t]{2}{*}{ Cínicos, M.Imagen } & 6,102 & 1,814 & $<, 0001$ & $\mathrm{~s}$ \\
\hline Clinicos, Normales & $-4,612$ & 928 & $<, 0001$ & s & & $-4,545$ & 1,118 & $<, 0009$ & s \\
\hline Clinicos, Rta. Inc. & $-6,104$ & 1,648 & $<, 0001$ & s & \multirow{2}{*}{$\begin{array}{l}\text { Clínicos, Rta.Inc. } \\
\text { M-imagen, Normales }\end{array}$} & $-6,109$ & 1,794 & $<, 0001$ & $s$ \\
\hline M.Imagen, Normales & $-11,632$ & 1,179 & $<, 0001$ & $s$ & & $-10,647$ & 1,647 & $<, 0001$ & s \\
\hline \multirow{2}{*}{$\begin{array}{l}\text { M.tmagen, Rta.Inc. } \\
\text { Normales, Rta.Inc. }\end{array}$} & $-\$ 3,123$ & 1,802 & $<, 0001$ & s & \multirow{2}{*}{$\begin{array}{l}\text { M.Imagen, Rta.Inc. } \\
\text { Normales, Rta.Inc. }\end{array}$} & $-12,211$ & 2,163 & $<, 0001$ & $\mathrm{~s}$ \\
\hline & $-1,492$ & 1,500 & .0513 & & & $-1,564$ & $\uparrow, 625$ &, 0592 & \\
\hline
\end{tabular}

Nota ${ }^{\mathrm{a}} \mathrm{m}=$ mujeres; $\mathrm{v}=$ Varones; $\mathrm{s}=$ significativo.

Cuando hemos realizado el análisis a través de su sexo diferente, hemos podio apreciar el hecho de que esta variable no discrimina adecuadamente (al 5\% de n.c.) los individuos que contestan, desde la normalidad, de forma sincera y honesta de aquellos otros que lo hacen sin tener en cuenta la información que contienen los ítemes. No ocurre lo mismo cuando se aúnan los sexos. 


\section{Diseño de la hoja de perfil según la estrategia del MMPI-2}

\section{El diseño tradicional del MMPI}

Tradicionalmente, la estrategia adoptada por la técnica del MMPI para mostrar el perfil de un determinado tipo de personalidad, reflejada en la hoja de perfil, ha sido a través de las puntuaciones directas y su equivalencia con las típicas $T$, cuya puntuación media es de 50 y su desviación estándar de 10.

Cuando contemplamos el diseño del perfil del MMPI (y también del MMPI-2), podemos apreciar que se configura teniendo en cuenta que las puntuaciones directas aparezcan en el interior del cuadro y tengan su correspondencia en puntuaciones típicas $T$ en ambos laterales de la hoja de perfil. Esta configuración es idéntica para cualquiera de los distintos grupos de Escalas (Básicas, Suplementarias o de Contenido). El punto de corte de las distintas variables pasó de 70T, en el MMPI, a 65T, en el MMPI-2.

Igualmente, el diseño tradicional muestra los valores de las puntuaciones directas de todas y cada una de las variables (expuestas en el interior de el Cuadro), en el mismo sentido que las expresadas por las puntuaciones $\mathrm{T}$; es decir, incrementando la puntuación de abajoarriba. Pero este mismo diseño muestra, referente a este aspecto y solamente en los varones, una excepción a través de la variable Masculinidad-Feminidad (Mf).

Esta Hoja de perfil, en su correspondiente apartado de las Escalas de Validez y Escalas Cínicas (existen otras hojas referentes a las Escalas Suplementarias y de Contenido) se muestran siempre en el mismo orden (de izquierda a derecha), incluso para su preferencia de interpretación: primero las Escalas de Validez (L, F y K) y en segundo lugar las 10 Escalas Clínicas. 
Nuestra propuesta de diseño, en la Hoja de Perfil, para algunas de las Escalas e Índices de Validez

En nuestra baremación de las distintas variables y Escalas indicadoras de Validez investigadas hemos mantenido escrupulosamente el mismo diseño referente a las puntuaciones directas, típicas y los mismos puntos de corte del MMPI-2. Nada se va a cambiar en la Hoja de perfil, excepto el sentido en sus puntuaciones directas de algunas variables (igual que en el perfil tradicional del MMPI de la variable $\mathrm{Mf}$ en los varones) en donde las puntuaciones directas inferiores se muestran a partir de la parte superior de la Hoja de perfil. Es decir, las puntuaciones directas más bajas se corresponden con las puntuaciones más elevadas en sus puntuaciones típicas $\mathrm{T}$, y viceversa.

En nuestra investigación, hemos seguido los criterios expuestos por Butcher, Dahlstrom, Graham, Tellegen y Kaemer. (1989) y Greene $(1997,2000)$ en sus trabajos al estudiar y analizar las distintas variables de validez indicadoras de "defensividad" o "fingimiento positivo", de tal forma que la indicación de este "fingimiento positivo" siempre se reflejase en la parte inferior de la Hoja de perfil (puntuaciones significativas $(1.5 \mathrm{DE})$ por debajo de $35 \mathrm{~T})$, mientras que la indicación de "fingimiento negativo" se reflejara en la parte superior de la Hoja (puntuaciones significativas (1.5 DE) superiores a 65T). Que el fingimiento positivo se muestre en las puntuaciones inferiores de la Hoja de perfil, o el fingimiento negativo se aprecie en la parte superior de la hoja, se debe a las investigaciones de Greene (1997, notas de las p. 203 y 204), en donde se muestran invertido los valores en las variables: $\mathrm{L}, \mathrm{K}$, ODecp, $\mathrm{S}$ y Wsd; es decir sus valores crecientes se especifican de arriba-abajo y no de abajo-arriba como en la mayoría de las variables en el diseño tradicional del MMPI (excepto Mf). 
Con esto intentamos conseguir los objetivos siguientes:

- Que, de una simple mirada a la Hoja de Perfil, pudiéramos apreciar que todas las puntuaciones de la parte superior del perfil (> 65T) fueran indicadoras de "fingimiento negativo"y/ o inconsistencia en sus respuestas, por el que el sujeto ha intentado mostrarse de una forma negativa (mala imagen, exagerando su sintomatología, hacerse el enfermo, etc.).

- Igualmente, todas las puntuaciones de la parte inferior del perfil $(<40 \mathrm{~T})$ fueran indicadoras de "fingimiento positivo" y/o consistencia en sus respuestas, (buena imagen, ajustado y equilibrado psicológicamente, minimizando su sintomatología, defensividad, etc.).

- Todas aquellas puntuaciones que se encontraran entre estos dos "puntos de corte", pudiéramos considerar como de "normalidad" en la que el sujeto ha contestado de una forma coherente, honesta y fiable.

La propuesta de esta "hoja de perfil" tiene una finalidad eminentemente práctica para que pudieran quedar reflejadas, de una forma contrastada, las numerosas escalas analizadas y cómo se presentan para cada uno de los grupos de la muestra.

Cambio de sentido de las variables tradicionales del MMPI: $L$ y $K$

¿Por qué cambiamos el sentido a estas variables?. Toda las investigaciones demuestran que las puntuaciones elevadas de estas dos variables se corresponden con un perfil de fingimiento positivo, en donde el sujeto intenta mostrar lo mejor de sí mismo, dando una buena imagen y expresando los aspectos favorables de su personalidad. Cuando nos encontramos con puntuaciones bajas, las implicaciones psicológicas de estas variables, efectivamente, cambian de sentido; el sujeto intenta mostrar su lado negativo, exagerando su sintomatología, intentando presentar el lado más negativo y desfavorable de sí mismo fingiéndose de una forma negativa. 
Cuando hemos realizado el cambio de sentido, hemos seguido la misma pauta para la Escala Clínica Masculinidad-Feminidad del MMPI en su baremo con los varones (véase cómo se ha hecho en la Cuadro 7).

\section{Cuadro 7}

Equivalencias de las puntuaciones típicas de las Escalas $L$ y $K$ al cambiar el sentido de sus puntuaciones directa

\begin{tabular}{|c|c|c|c|c|c|c|c|}
\hline \multicolumn{4}{|c|}{ Mujeres } & \multicolumn{4}{|c|}{ Varones } \\
\hline Típ/L & $\mathrm{PD} / \mathrm{L}$ & Típ/K & $\mathrm{PD} / \mathrm{K}$ & Típ/L & $\mathrm{PD} / \mathrm{L}$ & Típ/K & $\mathrm{PD} / \mathrm{K}$ \\
\hline 70 & 0 & 83 & 0 & 70 & 0 & 81 & 0 \\
\hline 66 & 1 & 80 & 1 & 66 & 1 & 79 & 1 \\
\hline 62 & 2 & 78 & 2 & 61 & 2 & 77 & 2 \\
\hline 57 & 3 & 76 & 3 & 57 & 3 & 75 & 3 \\
\hline 53 & 4 & 74 & 4 & 53 & 4 & 73 & 4 \\
\hline 49 & 5 & 71 & 5 & 49 & 5 & 71 & 5 \\
\hline 44 & 6 & 69 & 6 & 45 & 6 & 69 & 6 \\
\hline 40 & 7 & 67 & 7 & 41 & 7 & 67 & 7 \\
\hline 36 & 8 & 64 & 8 & 37 & 8 & 64 & 8 \\
\hline 32 & 9 & 62 & 9 & 33 & 9 & 62 & 9 \\
\hline 27 & 10 & 60 & 10 & 29 & 10 & 60 & 10 \\
\hline 23 & 11 & 58 . & 11 & 25 & 11 & 58 & 11 \\
\hline 19 & 12 & 55 & 12 & 21 & 12 & 56 & 12 \\
\hline 14 & 13 & 53 & 13 & 17 & 13 & 54 & 13 \\
\hline 10 & 14 & 51 & 14 & 13 & 14 & 52 & 14 \\
\hline 6 & 15 & 49 & 15 & 9 & 15 & 50 & 15 \\
\hline & & 46 & 16 & & & 47 & 16 \\
\hline & & 44 & 17 & & & 45 & 17 \\
\hline & & 42 & 18 & & & 43 & 18 \\
\hline & & 39 & 19 & & & 41 & 19 \\
\hline & & 37 & 20 & & & 39 & 20 \\
\hline & & 35 & 21 & & & 37 & 21 \\
\hline & & 33 & 22 & & & 35 & 22 \\
\hline & & 30 & 23 & & & 33 & 23 \\
\hline & & 28 & 24 & & & 30 & 24 \\
\hline & & 26 & 25 & & & 28 & 25 \\
\hline & & 23 & 26 & & & 26 & 26 \\
\hline & & 21 & 27 & & & 24 & 27 \\
\hline & & 19 & 28 & & & 22 & 28 \\
\hline & & 17 & 29 & & & 20 & 29 \\
\hline & & 14 & 30 & & & 18 & 30 \\
\hline
\end{tabular}

Pero, ¿qué implicaciones tiene esto para la interpretación tradicional de estas Escalas (L y K) en el MMPI?: 
Cuando las interpretaciones se hacen a través de las puntuaciones directas, esto no implica cambio alguno. Es decir, una puntuación directa en cualquiera de estas dos escalas ( $\mathrm{L}$ y $\mathrm{K}$ ) siempre indicarán lo mismo, tanto en el MMPI (o MMMPI-2) tradicional como en nuestro diseño.

Cuando las interpretaciones se realizan a través de las puntuaciones típicas $T$, es necesario tener cuidado, ya que un cambio de sentido en las puntuaciones directas (de nuestro diseño), implica un cambio en la dirección de sus puntuaciones típicas T. Es decir, en el MMPI (o MMPI-2) una puntuación típica de 65T (1.5 DE), ahora se corresponde con una puntuación de $35 \mathrm{~T}$ (1.5 DE, pero por abajo), y su interpretación debe ser idéntica.

Respecto de nuestra variable investigada, la Escala Superlativa, "S"3

Tradicionalmente, en la Hoja de perfil del MMPI/MMPI-2, las puntuaciones directas, insertas en el interior de la hoja de perfil y exceptuando la variable clínica Mf en las mujeres, se han expresado en el sentido de "menor a mayor" coincidiendo con el mismo sentido de las puntuaciones $T$. Es decir, las puntuaciones directas menores, o más bajas, se correspondían con las puntuaciones menores en las puntuaciones $T$, e igualmente, las puntuaciones directas elevadas se correspondían con las puntuaciones $T$ elevadas.

En nuestro diseño, pretendiendo exponer en la Hoja de perfil del MMPI-2 las puntuaciones significativas de "fingimiento positivo" en la parte inferior, y el "fingimiento negativo" en la parte superior, hemos tenido que acomodar el sentido de las puntuaciones directas, en la $\mathrm{S}$ al sentido dado por el autor: una mayor puntuación directa se corresponde con un mayor "fingimiento positivo", y viceversa, una puntuación menor se corresponde con una ausencia de tal fingimiento.

Por ello, la escala $\mathrm{S}$ va a presentarse con sus puntuaciones directas más elevadas en la zona inferior de la Hoja de perfil del MMPI-2,

Otro tanto ocurre con otras variables como Odecp y Wsd. 
al modo como se encuentran, en el perfil tradicional del MMPI-2, la variable Mf para las mujeres (cambiando el sentido de las puntuaciones directas).

Puntos de corte en la zona inferior y superior del perfil (puntuaciones T)

Tradicionalmente, en la Hoja de perfil del MMPI-2, se considera como zona de "normalidad" entre 1,00 desviación estándar por encima y por debajo de la puntuación media $(M=50)$ ya que por condicionamientos puramente estadísticos las puntuaciones típicas presentan una puntuación media de 50 y una desviación estándar de 10. La significación siempre se planteará, en puntuaciones típicas $T$, con 1,00 desviación estándar por encima y/o por debajo de la media ( $1 \mathrm{DE})$ convirtiéndose en $60 \mathrm{~T}$ y/o $40 \mathrm{~T}$ respectivamente.

El MMPI tradicional mantenía como punto de corte, significativo de presencia del factor en cuestión, en 70T, esto significaba dos desviaciones estándar a partir de la puntuación media. En la reestandarización, MMPI-2, se ha rebajado en $0.5 \mathrm{DE}$ el punto de corte significativo de incidencia del factor, quedando en $65 \mathrm{~T}$. En nuestro diseño seguimos manteniendo estos mismos parámetros de puntos de corte del MMPI-2 en las puntuaciones superiores; es decir, $65 \mathrm{~T}$ para la significatividad para cualquier tipo de índice o Escala.

No obstante, nuestro diseño para las Escalas e Índices de Validez, propone, siguiendo la misma filosofía estadística, la existencia de un punto de corte por debajo de la media, especificado en $35 \mathrm{~T}$ correspondientes a $1.5 \mathrm{DE}$ por debajo de la media (Cuadro 8).

Con todo ello, y siempre teniendo la perspectiva de poder apreciar de un "golpe de vista" la existencia de fingimiento, pretendemos exponer tres zonas fundamentales en la Hoja de Perfil del MMPI-2:

- Zona de inconsistencia y/o fingimiento negativo significativo: puntuaciones $=65 \mathrm{~T}$ 
- Zona de normalidad: puntuaciones entre 35T - 65T.

- Zona de fingimiento positivo: significativo: Puntuaciones $=35 \mathrm{~T}$.

\section{Cuadro 8}

Puntos de corte de las Escalas de Validez y de la Superlativa $S$

\begin{tabular}{|ccccccccccccccc|}
\hline \multicolumn{2}{|c}{ Escalas/Punt. } & T & $20 \mathrm{~T}$ & \multicolumn{2}{c}{$35 \mathrm{~T}$} & \multicolumn{2}{c}{$50 \mathrm{~T}$} & \multicolumn{2}{c}{$65 \mathrm{~T}$} & \multicolumn{2}{c|}{$80 \mathrm{~T}$} & \multicolumn{2}{c|}{ 100T } \\
\hline & Varón & Mujer & Varón & Mujer & Varón & Mujer & Varón & Mujer & Varón & Mujer & Varón & Mujer \\
VRIN & - & - & 3 & 3 & 8 & 8 & 13 & 12 & 17 & 16 & 24 & 23 \\
TRIN-F & - & - & - & - & - & - & 7 & 7 & 5 & 5 & 1 & 1 \\
TRIN-V & - & - & - & - & 10 & - & - & 12 & 15 & 15 & 18 & 16 \\
L & 12 & 12 & 7 & 9 & 5 & 5 & 1 & 1 & - & - & - & - \\
F & - & - & 2 & 2 & 8 & 6 & 17 & 19 & 26 & 30 & 38 & 46 \\
Fb & - & - & - & - & 4 & 4 & 12 & 10 & 19 & 16 & 29 & 23 \\
K & 29 & 28 & 20 & 21 & 15 & 15 & 7 & 7 & 1 & 1 & - & - \\
$\mathrm{S}$ & 48 & 47 & 35 & 36 & 24 & 24 & 11 & 12 & 0 & 2 & - & - \\
\end{tabular}

Teniendo en cuenta este pequeño cambio de diseño en la Hoja de perfil, podemos apreciar "de un golpe de vista" en las gráficas 2 y 3 cómo las Escalas de Validez y la Escala Superlativa S la "buena imagen" presentan sus puntuaciones por debajo de la línea media ( $T$ $=50$ ), y cuando quieren expresar la "mala imagen" de sí mismo todas las puntuaciones de las escalas aparecen superando la línea media $(T=50)$ de la hoja de perfil.

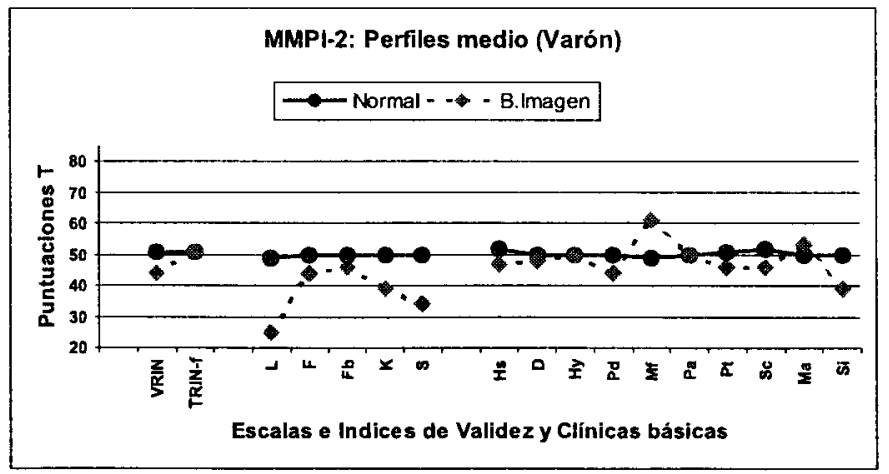

Figura 2. Comportamiento del perfil al comparar el grupo de sujetos Normales con los que muestran Buena Imagen (perfiles medios). 
En la Figura 2, podemos observar lo difícil que resulta apreciar una "buena imagen". Por parte de las variables que detectan consistencia o coherencia en sus respuestas, observamos que tanto la VRIN como la TRIN se comportan dentro de la normalidad (efectivamente, el sujeto ha contestado la prueba dando una imagen de sí mismo, tanto favorable, como desfavorable -ver gráfica 3- de una manera "adecuadamente coherente". Si fijamos nuestra atención en las escalas Clínicas básicas, donde más claramente se percibe la falsificación es en el perfil de los sujetos que coherentemente han mostrado su "mala imagen", pero tenemos grandes dificultades (ver grafía 2) cuando queremos detectar la "buena imagen". Hemos de centrar nuestra atención expresamente en las dos escalas L y S para poder ayudarnos a detectar la defensividad del sujeto a través de su buena imagen.

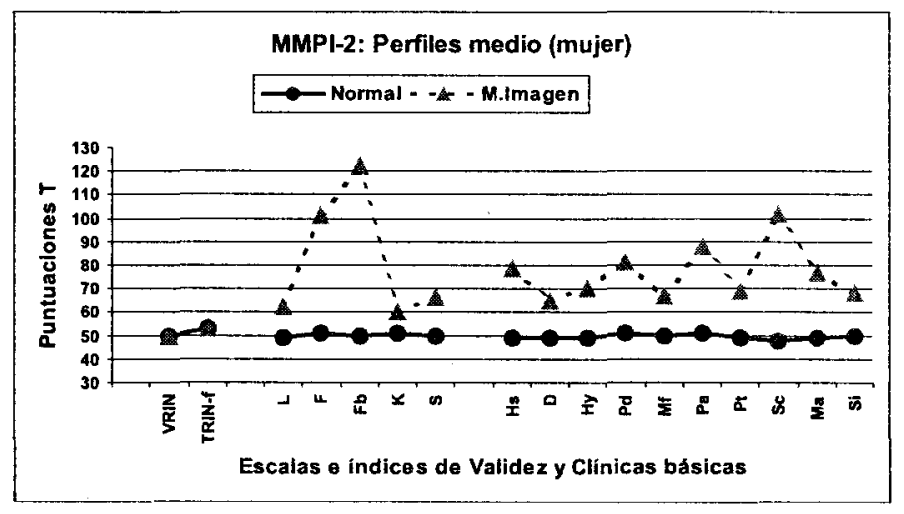

Figura 3. Comportamiento del perfil al comparar el grupo de sujetos Normales con los que muestran Mala Imagen (perfiles medios).

\section{Discusión}

Greene (1997) hace una revisión de las diferentes estrategias adoptadas para evaluar el fingimiento y la defensividad a través de los diversos cuestionarios existentes en la evaluación de la personalidad. Cuando Meehl y Hathaway (1946) elaboraron el MMPI estaban 
convencidos de la posibilidad de falseamiento de la prueba, de que alguien podría obtener beneficios personales a través de esta falsificación. Para ello elaboraron dos tipos de categorías generales en las que estaban incluidos los falsificadores: la considerada por ellos como "fingimiento" (fingirse mal) y la de "defensividad" ("fingirse bien"). Estos autores consideraron tres estrategias posibles para evaluar el fingimiento y la defensividad:

1. La inconsistencia (o "incoherencia") de respuestas para los ítemes de contenido semejante. Con esta estrategia, el clínico propone al sujeto una serie de ítemes con la oportunidad y posibilidad de poder distorsionar fácilmente su respuesta y observar, posteriormente, los resultados de sus respuestas para los ítemes redactados de idéntica forma, tanto en sentido positivo como en negativo. El Cuestionario del MMPI original presenta 16 ítems repetidos. Los resultados aportaron un gran número de respuestas incoherentes, sugiriendo a los autores, que las personas se mostraron incapaces o poco dispuestas a responder de forma coherente. Por ello, los mismos autores no estaban muy convencidos de que este tipo de estrategia "funcionara" adecuadamente. En la reestandarización del MMPI estos 16 ítems de redacción idéntica, fueron eliminados y se elaboraron dos nuevas escalas denominadas "Inconsistencia de Respuestas Variable (VRIN) e "Inconsistencia de Respuestas Verdadero" (TRIN) que veremos más adelante.

2. Los ítemes sumamente convenientes pero infrecuentemente atribuidos. Con esta segunda estrategia se elaboraron una serie de ítems que fueran extremadamente deseables de contestar en una dirección determinada pero que, justamente en esta misma dirección de la respuesta, fuese muy probablemente falso. Estos ítems atentan contra las cualidades humanas de educación, responsabilidad, honestidad, etc., de las cuales, en todos presuponemos una tendencia socialmente deseable y natural. Si una persona se atribuye un gran número de estos ítems, la probabilidad de que las respuestas no sean 
sinceras es muy alta. La escala L (Mentira) fue elaborada con este objetivo, indicando con ello un grado de "defensividad" de la persona. La escala $F$ (infrecuencia) se elaboró con arreglo a una variante de esta misma segunda estrategia con la finalidad de valorar actitudes de respuestas al test. Los ítems para esta escala $F$ fueron inicialmente seleccionados porque eran contestados con una relativa baja frecuencia por la mayor parte del grupo de población normal evaluado con el MMPI. Es decir, si una persona se atribuye un gran número de ítemes de esta escala $F$, esta persona responde de forma atípica con referencia a su grupo normativo. Esta misma estrategia fue adoptada por el MMPI-2 y ampliada con la elaboración de la escala Fb (F posterior) que hace referencia a la segunda parte de la prueba.

3. Ítems empíricamente identificados. Una última y tercera estrategia para detectar el fingimiento y la defensividad evaluada por el MMPI fue la aportada por estos mismos autores, Meehl y Hathaway (1946), elaborando un procedimiento empírico para identificar los items de los que se obtienen diferentes respuestas con dos grupos seleccionados de personas: aquellos que contestan de forma sincera y aquellos otros que son instruidos para falsear la prueba. La escala de Disimulación de Gough (Ds, 1954) y su "revisada" (Ds-r, 1957), se encuentran basadas en esta estrategia. Meehl y Hathaway (1946), adoptaron una variante de esta tercera estrategia desarrollando la escala K (correctora), elaborada para diferenciar pacientes internados con perfiles normales de individuos, supuestamente normales, con perfiles anormales. Posteriormente determinaron las diferentes proporciones de $\mathrm{K}$ que deben ser añadidas a determinadas escalas Clínicas para maximizar la discriminación entre el grupo criterio y el grupo normativo.

El hecho de haberse tomado, para la Escala Superlativa S de Butcher y Han (1995), como criterio la selección de sujetos varones (graduados universitarios, raza blanca, "triunfadores" en la vida y con un buen nivel socioeconómico) para pilotos de líneas aéreas para 
elaborar la escala superlativa S, está suscitando todo tipo de controversias acerca del género, de la deseabilidad social y de la franca sinceridad de los sujetos. No está claro si S posee el mismo grado de independencia de psicopatología que $\mathrm{K}$, pero parece ser al menos tan sensible a las actitudes autofavorables frente a las actitudes desfavorables de los examinados (Greene, 2000).

La escala $S$ parece haber discriminado adecuadamente aquellos estudios con el MMPI-2 (Baer, Wetter, Nichols, Greene y Berry, 1995; Bagby, Nicholson, Buis, Radovanovic y Fiddler, 1999) en los que se comparan la habilidad de diferentes escalas de infravaloración cumplimentadas siguiendo una determinada estrategia de presentar una imagen favorable de sí mismo de aquellos otros sujetos que han contestado al cuestionario de forma estándar (sincera y honestamente).

Para Nichols (2002), la interpretación de S puede ser reforzada teniendo en cuenta sus puntuaciones dentro del contexto de las obtenidas en L y Odecp (Escala de Fingimiento Positivo o de Otro Engaño, de Nichols y Greene, 1991) cuando sus puntuaciones respectivas se muestran elevadas; y con F, Fb, Fp (Escala Psiquiátrica de Arbisi y Ben-Porath, 1995), Ds-r (Escala revisada de Disimulación de Gough, 1957), y F-K (Indice de Gough, 1947, 1950) cuando sus puntuaciones son bajas.

Nuestros resultados son plenamente coincidentes con los citados por Nichols (2002). Para ayudarnos de una mejor interpretación diagnóstica, podemos acompañarnos de los resultados elevados de las variables $\mathrm{K}, \mathrm{L}$ y ODecp y, de forma contraria, de las puntuaciones más bajas ofrecidas por F, las escalas de Disimulación de Gough (Ds y Ds-r) y la F-K que correlaciona de forma altamente negativa como se puede apreciar en la Tabla de correlaciones.

Para Nichols (2002), las puntuaciones hasta 60T-70T sugieren. bien una presentación de sí mismo totalmente favorable como resul- 
tado de actitudes propias ingenuas, comprensión intelectual limitada y auto-vigilancia, o bien miedo de cómo serán considerados y utilizados los resultados del test. En estos niveles son infrecuentes los intentos deliberados de engañar y es posible ayudarse de las puntuaciones en otras escalas como las Odecp (Escala de Fingimiento Positivo o de Otro Engaño, de Nichols \& Greene, 1991) y las Wsd (Escala de Deseabilidad Social de Wiggins, 1959) ${ }^{4}$, pero especialmente cuando los valores de Wsd se muestran superiores a los de ODecp. Solamente debemos referir un perfil distorsionado por la infravaloración cuando las puntuaciones $\mathrm{T}$ alcanzan los valores superiores a 70 , afectando de manera importante a las Escalas de Contenido del MMPI-2 que se reflejan ligeramente más bajas.

\section{Conclusiones}

Resumiendo de forma breve nuestras conclusiones, a través de los resultados obtenidos, son las siguientes:

- Las altas puntuaciones directas de la Escala Superlativa (S) de Butcher y Han (1995) identifican, de manera significativa, a las personas que intentan ofrecer una imagen favorable de sí mismo, mostrándose extremadamente virtuosas, equilibradas y altamente ajustadas psicológicamente.

- Esta variable $\mathrm{S}$ ha presentado una alta discriminación en sus valores medios, estadísticamente significativos $(p<.0001)$, con el resto de los grupos aquí investigados. Cuando el análisis se ha realizado separadamente por sexos, podemos apreciar que los sujetos normales, tanto varones como mujeres, no se han diferenciado estadísticamente de aquellos otros que contestan sin tener en cuenta la información contenida en los ítems (Rptas. Inconsit.).

4 En artículos sucesivos se irán publicando nuestros resultados con estas escalas. 
- Las puntuaciones directas elevadas de S no discriminan suficientemente a los sujetos que contestan intentando mostrar una imagen favorable de sí mismos de aquellos otros que contestan a todos los ítemes del Cuestionario como "falso".

- Para una interpretación diagnóstica más precisa, podríamos ayudarnos de las aportaciones de L, K, ODecp y Wsd con las que se obtuvieron correlaciones altamente positivas y han demostrado evaluar el mismo concepto.

- El 90\% de los sujetos que presentaron esta imagen favorable de sí mismos contestaron a la mitad de los ítemes correspondientes a esta escala de forma poco honesta y sincera, obteniendo una puntuación $\mathrm{PD}=25$ (puntuación media $=36.33$ ), semejante al $50 \%$ de los sujetos normales que se mostraron sinceros y honestos en sus respuestas $\mathrm{PD}=24$ (puntuación media $=23.89)$ o al $25 \%$ de sujetos clínicos, también sinceros, que obtuvieron una puntuación $\mathrm{PD}=24$ (puntuación media $=19,316$ ).

- El "punto de corte" en nuestra baremación quedó establecido en una $\mathrm{PD}=37(34 \mathrm{~T})^{5}$ para los varones, y de $\mathrm{PD}=36(34 \mathrm{~T})$ para las mujeres, significando con ello que a partir de estas puntuaciones podemos considerar que el sujeto intenta mostrar su imagen favorable.

\section{Referencias}

Avila, A. y Jiménez, F. (1999). Adaptación española del Inventario Multifásico de Minnesota-2. Madrid: TEA.

Baer, R. A., Wetter M. W. y Berry D. (1995). Effects of information about validity scales on underreporting of symptoms on the MMPI-2: An analogue investigation. Assessment, 2(2), 189-200.

Baer, R. A., Wetter, M.W., Nichols, D. S., Greene R. L. y Berry, D. T. R. (1995). Sensivity of MMPI-2 validity scales to underreporting of symptoms. Psychological Assessment: A Journal of Consulting and Clinical Psychology, 7, 419-423. 
Bagby, R., Rogers, R., Buis, T., Nicholson, R., Cameron, S., Rector, N., Schuller, D. y Seeman, M. (1997). Detecting feigned depression and schizophrenia on the MMPI-2. Journal of Personality Assessment, 68(3), 650-664.

Bagby, R. M., Nicholson, R. A., Buis, T., Radovanovic H. y Fiddler, B. J. (1999). Defensive responding on the MMPI-2 in family custody and access evaluations. Psychological Assessment: A Journal of Consulting and Clinical Psychology, 11, 24-28.

Butcher, J., y Han, K. (1995). Development of an MMPI-2 scale to assess the presentation of self in a superlative manner: The $S$ scale. En J. N. Butcher y C.D. Spielberger (Eds.), Advances in personality assessment (vol. 10) (pp. 25-50). Hillsdale, NJ: LEA Press.

Butcher, J. N., Dahlstrom, W., Graham, J., Tellegen, A. y Kaemer, B. (1989). Manual for the reestandaridized Minnesota Multiphasic Personality Inventory: MMPI-2. Minneapolis University of Minnesota Press.

Dahlstrom, W., Welsh, G. y Dahlstrom, L. E. (1975). An MMPI handbook. Volume II.: Research developments and interpretations (Ed. Revisada). Minneapolis: University of Minnesota Press.

Dahlstrom, W. G., Lachar, D. y Dahlstrom, L. E. (1986). MMPI patterns of American minorities. Minneapolis. University of Minnesota Press.

Graham, J. (2000). MMPI-2, assessing personality and psychopathology (3ra. ed.). Oxford: University Press.

Graham, J., Watts, D. y Timbrook, R. (1991). Detecting fake-good and fake-bad MMPI-2 profiles. Journal of Personality Assessment, $57(2), 264-277$.

Greene, R. L. (1997). Assessment of malingering and defensiveness by multiscale inventories. En R. Rogers (Ed.), Clinical assessment of malingering and deception (2da. ed.) (pp. 169-207). Nueva York: Guilford Press.

Greene, R. L. (2000). The MMPI-2. An interpretative manual (2da. ed.). Boston: Allyn and Bacon. 
Gynther, M. D., Alman, H. y Warbin, W. (1973). Interpretation of uninterpretable Minnesota Multiphasic Personality Inventory profiles. Journal of Consulting and Clinical Psychology, 40, 7883.

Jiménez, F. y Sánchez, G. (2003). Evaluación psicológica forense.-4. Contribución de las técnicas de Minnesota y Millon. Amarú ediciones (en prensa).

Jiménez, F. y Sánchez, G. (2001). La contribución de las escalas Obvio-sutil del MMPI-2 a la detección del fingimiento. Revista Iberoamericana de Diagnóstico y Evaluación Psicológica, 11(1): 111130.

Mckinley, J. C. y Hathaway, S. R. (1948). A multiphasic personality schedule (Minnesota). VI. The K scale. Journal of Consulting Psychology, 12, 20-31.

Meehl, P. E. y Hathaway, S. R. (1946). The K factor as a suppressor variable I: the MMPI. Journal of Applied Psychology, 30, 525564.

Nichols, D. S. (2002). Claves para la evaluación con el MMPI-2.Madrid: TEA.

Rogers, R. (1990). Development of a new classificatory model of malingering. Bulletin of the American Academy of Psychiatry and the Law, 18(3), 323-333.

Sánchez, G. (2002). Sensibilidad de las escalas e indicadores de Validez en el perfil de personalidad del MMPI-2. Tesis doctoral. Universidad de Salamanca, Salamanca.

Sánchez, G., Jiménez, F., Avila, A y Merino, V. (1999). Psicopatología y Fiabilidad: un análisis comparativo de las escalas de Validez entre el MCMI-II y el MMPI-2. Revista Iberoamericana de Diagnóstico y Evaluación Psicológica, 7(1), 115-125. 


\section{Anexo \\ Escala Superlativa (S) de Butcher y Han (1995)}

15- Trabajo bajo una tensión muy grande.

50- Con frecuencia he tenido que recibir órdenes de personas que sabían menos que yo.

58- Pienso que una gran mayoría de la gente exagera sus desgracias para lograr la simpatía y ayuda de los demás.

76- Cuesta mucho trabajo convencer a la mayoría de la gente de la verdad.

81- Creo que la mayoría de la gente mentiría con tal de conseguir lo que quiere.

87- Me he enfrentado a problemas con tantas opciones de solución que me ha sido imposible llegar a una decisión.

89- Los conflictos más graves que tengo son conmigo mismo.

104- La mayoría de la gente es honrada por temor a ser descubierta.

110- Para no perder un beneficio o ventaja, la mayoría de la gente utilizaría medios algo injustos.

120- Frecuentemente encuentro necesario defender lo que es justo.

121- Nunca me he entregado a prácticas sexuales fuera de lo común.

123- Si pudiera entrar en un espectáculo sin pagar y estuviera seguro de no ser visto, probablemente lo haría.

148- Nunca en mi vida me he sentido mejor que ahora.

154- Siento miedo cuando miro hacia abajo desde un lugar alto.

184- Muy pocas veces sueño despierto.

194- Nunca he tenido erupciones en la piel que me hayan preocupado.

196- Frecuentemente me encuentro preocupado por algo.

205- Alguno de mis familiares tienen costumbres que me molestan y me irritan muchísimo.

213- Me enfado fácilmente pero se me pasa pronto.

225- Mi manera de hacer las cosas tiende a ser mal interpretada por los demás. 
264- He abusado de bebidas alcohólicas.

279- Me niego a participar en algunos juegos porque no soy hábil en ellos.

284- Pienso que casi todo el mundo mentiría para evitarse problemas.

290- Me preocupan el dinero y los negocios.

302- Pierdo fácilmente la paciencia con la gente.

337- En las reuniones sociales o fiestas es más probable que me siente solo o con una persona antes que reunirme con un grupo.

341- En ocasiones mi mente parece funcionar más lenta que de costumbre.

346- Con frecuencia me he encontrado con personas supuestamente expertas, que no resultaron ser mejores que yo.

352- Normalmente la gente pide para sus propios derechos más respeto que el que está dispuesto a conceder a los demás.

373- Nunca hablo de las cosas malas que he hecho.

374- La mayoría de la gente utilizaría medios algo injustos para salir adelante en la vida.

403- Frecuentemente la gente ha malinterpretado mis intenciones cuando trataba de corregirles o ayudarles.

420- Me pone nervioso tener que esperar.

423- Con frecuencia me esfuerzo para triunfar sobre alguien que se enfrenta a mí.

428- Varias veces he cambiado de modo de pensar con respecto a mi trabajo.

430- Frecuentemente me lamento por tener mal genio o ser tan gruñón.

433- Cuando estoy en una situación difícil sólo digo aquella parte de la verdad que probablemente no me va a perjudicar.

442- Debo admitir que a veces he estado demasiado preocupado por algo sin importancia.

445- Frecuentemente he trabajado bajo órdenes de personas que parecen haber arreglado las cosas de tal modo, que ellas son las que reciben el reconocimiento de una buena labor y, en cambio, son capaces de atribuir los errores a sus subordinados.

449- Algunos de mis familiares tienen muy mal genio. 
La Escala Superlativa S de Butcher y Han (1995)

461 - Me pone de mal humor que la gente me meta prisas.

486- Soy muy terco (tozudo/a, insistente).

487- He disfrutado tomando drogas.

523- Me molesta mucho tener que hacer colas en los cines, restaurantes o acontecimientos deportivos.

534- Si pudiera comenzar mi vida de nuevo, no la cambiaría mucho.

538- De vez en cuando la mayoría de los maridos son infieles a sus esposas.

542- Me he enfadado tanto con alguien que he estado a punto de explotar.

545- Siempre tengo muy poco tiempo para hacer las cosas.

547- A menudo guardo y conservo cosas que probablemente nunca usaré.

560- Estoy satisfecho con la cantidad de dinero que gano. 